\title{
Synergistic community responses of plants and arbuscular mycorrhizal fungi to extreme droughts in a cold-temperate grassland
}

Wei $\mathrm{Fu}^{1}$, Baodong $\mathrm{Chen}^{1}$, Matthias Rillig${ }^{2}$, Wang $\mathrm{Ma}^{3}$, Chong $\mathrm{Xu}^{3}$, Wentao Luo ${ }^{3}$, Honghui $\mathrm{Wu}^{3}$, Zhipeng $\mathrm{Hao}^{1}$, Hui $\mathrm{Wu}^{3}$, Aihua Zhao ${ }^{1}$, Qiang $\mathrm{Yu}^{4}$, and Xingguo $\mathrm{Han}^{3}$

${ }^{1}$ Research Centre for Eco-Environmental Sciences Chinese Academy of Sciences

${ }^{2}$ Freie Universität Berlin

${ }^{3}$ Affiliation not available

${ }^{4}$ National Hulunber Grassland Ecosystem Observation and Research Station, Institute of Agricultural Resources and Regional Planning, Chinese Academy of Agricultural Sciences

November 26, 2020

\begin{abstract}
Mutualistic associations between plants and arbuscular mycorrhizal (AM) fungi may have profound influences on their response to climate changes. Existing theories evaluate the effects of interdependency and environmental filtering on plant-AM fungal community dynamics separately; however, abrupt environmental changes such as climate extremes can provoke duo-impacts on the metacommunity simultaneously. Here, we experimentally tested the relevance of plant and AM fungal community responses to extreme drought (chronic or intense) in a cold temperate grassland. Irrespective of drought intensities, plant species richness and productivity responses were significantly and positively correlated with AM fungal richness and also served as best predictors of AM fungal community shifts. Notably, the robustness of this community synergism increased with drought intensity, likely reflecting increased community interdependence. Network analysis showed a key role of Glomerales in AM fungal interaction with plants, suggesting specific plant-AM fungal pairing. Thus, community interdependence may underpin climate change impact on plant-AM fungal diversity patterns in grasslands.
\end{abstract}

Synergistic community responses of plants and arbuscular mycorrhizal fungi to extreme droughts in a cold-temperate grassland

Author: Wei $\mathrm{Fu}^{1,2}$, Baodong Chen ${ }^{1,2}$, Matthias C. Rillig ${ }^{3,4}$, Wang $\mathrm{Ma}^{5,2}$, Chong $\mathrm{Xu}^{6,7}$, Wentao $\mathrm{Luo}^{5}$, Honghui $\mathrm{Wu}^{6}$, Zhipeng $\mathrm{Hao}^{1}$, Hui $\mathrm{Wu}^{1,2}$, Aihua Zhao ${ }^{1,2}$, Qiang $\mathrm{Yu}^{8}$, Xingguo $\mathrm{Han}^{9,}{ }^{2,5}$

${ }^{1}$ State Key Laboratory of Urban and Regional Ecology, Research Center for Eco-Environmental Sciences, Chinese Academy of Sciences, Beijing 100085, China

${ }^{2}$ University of Chinese Academy of Sciences, Beijing 100049, China

${ }^{3}$ Institute of Biology, Freie Universität Berlin, Berlin 14195, Germany

${ }^{4}$ Berlin-Brandenburg Institute of Advanced Biodiversity Research (BBIB), Berlin 14195, Germany

${ }^{5}$ Erguna Forest-Steppe Ecotone Research Station, Institute of Applied Ecology, Chinese Academy of Sciences, Shenyang 110164, China

${ }^{6}$ Ministry of Agriculture Key Laboratory of Crop Nutrition and Fertilization, Institute of Agricultural Resources and Regional Planning, Chinese Academy of Agricultural Sciences, Beijing 100081, China 
${ }^{7}$ State Key Laboratory of Grassland Agro-ecosystems, College of Pastoral Agriculture Science and Technology, Lanzhou University, Lanzhou 730020, China

${ }^{8}$ National Hulunber Grassland Ecosystem Observation and Research Station, Institute of Agricultural Resources and Regional Planning, Chinese Academy of Agricultural Sciences, Beijing 10008, China

${ }^{9}$ State Key Laboratory of Vegetation and Environmental Change, Institute of Botany, Chinese Academy of Sciences, Beijing 100093, China

Author contributions : BC, WF and MCR conceived the idea of this study. XH, HW and QY designed the field experiment. WM, CX, HW, QY and WL conducted the daily management of the field experiment and collected the plant data. WF, ZH, HW and AZ measured the soil physicochemical properties. WF performed AM fungi-related experiments, analyzed all the data and created all the figures. WF, BC, HW and MCR wrote the first draft of the manuscript, and all co-authors contributed substantially to the revisions.

Data availability : If the manuscript is accepted for publication, all the data supporting the results will be archived in Figshare and the data DOI will be included at the end of the article.

Running title : Community responses to extreme drought

Keywords : arbuscular mycorrhiza, mutualism, community interactions, belowground biodiversity, species coexistence, environmental filtering, climate change.

Correspondence should be addressed to

Baodong Chen :

Tel: +86-010-62849068;

Fax: +86-010-62923549;

E-mail: bdchen@rcees.ac.cn;

Mailing address: 18 Shuangqing Road, Haidian, Beijing 100085, China

\section{Honghui Wu}

Tel: +86-010-82108699;

Fax: +86-010-82106225;

E-mail: wuhonghui@caas.cn;

Mailing address: 12 Zhongguancun South Street, Haidian District, Beijing 100081, China

Article type : Letters

Number of words in the abstract : 154

Number of words in the main text : 4895

Number of references : 80

Number of figures : 5 Abstract

Mutualistic associations between plants and arbuscular mycorrhizal (AM) fungi may have profound influences on their response to climate changes. Existing theories evaluate the effects of interdependency and environmental filtering on plant-AM fungal community dynamics separately; however, abrupt environmental changes such as climate extremes can provoke duo-impacts on the metacommunity simultaneously. Here, we experimentally tested the relevance of plant and AM fungal community responses to extreme drought (chronic or intense) in a cold temperate grassland. Irrespective of drought intensities, plant species richness and productivity responses were significantly and positively correlated with AM fungal richness and also served as best predictors of AM fungal community shifts. Notably, the robustness of this community 
synergism increased with drought intensity, likely reflecting increased community interdependence. Network analysis showed a key role of Glomerales in AM fungal interaction with plants, suggesting specific plant-AM fungal pairing. Thus, community interdependence may underpin climate change impact on plant-AM fungal diversity patterns in grasslands.

\section{INTRODUCTION}

The ecology and evolution of plants and soil microbial communities are strongly interlinked and therefore considered as an interrelated cross-kingdom holobiont with pivotal importance in maintaining ecosystem stability (Bardgett \& van der Putten 2014; Vandenkoornhuyse et al. 2015; Yang et al . 2018). Among the plant-microbe interactions, the arbuscular mycorrhizal (AM) association is of particular importance. In this ubiquitous symbiosis, the fungi trade nutrients for carbon with their plant partners (Smith \& Read 2010; Kiers et al. 2011; Powell \& Rillig 2018). Within the partnership, both plants and AM fungi can functionally help their partner to resist a wide range of biotic and abiotic stresses (Smithet al. 2009; Delavaux et al. 2017; $\mathrm{Xu}$ et al . 2018). For instance, AM association could alleviate plant drought stress by altering hormonal profiles (Ruiz-Lozano et al. 2016), increasing water transport via up-regulating AM fungal aquaporin genes (Li et al. 2013), and stabilizing plant stoichiometric homeostasis via enhancing plant nutrient uptake (Bowles et al. 2018). Plants, in turn, would transfer a proportional amount of photosynthetic carbon to AM fungi (Kiers et al. 2011; Karlowsky et al. 2018). However, despite this functional interdependence, it remains largely unknown whether plant and AM fungal community responses are interrelated, and if so, how communities interact to respond to environmental changes. This lack of knowledge hampers our ability to understand ecosystem resistance and resilience, and to predict plant-AM fungal community dynamics under both current and future climate.

The community dynamics of both plants and AM fungi depend on dispersal limitation, abiotic filtering, and biotic interactions (Götzenbergeret al. 2012; Zobel \& Öpik 2014; Vályi et al. 2016). Translating these driving forces into specific processes, several hypotheses have been formulated to aid our understanding of plant-AM fungal community assembly mechanisms. However, these ideas focus on different driving forces separately, making them less informative under climate change. For instance, the passenger and driver hypotheses (Hart et al. 2001), which assume that plants can drive AM fungal community dynamics orvice versa , maybe impractical for community assembly shaped by environmental filtering (Davison et al . 2015; Van Geel et al. 2018). Likewise, the habitat hypothesis also has its drawbacks for not considering complex community interactions and feedbacks driven by biotic interactions (van der Heijden et al. 1998; Rillig et al . 2014; Neuenkamp et al. 2018). Beyond these hypotheses, plants and AM fungi show a range of conflicting community interaction patterns, from positive (van der Heijden et al. 1998; Hiiesalu et al. 2014), to independent (Öpik et al. 2010; Horn et al. 2017), and negative (Antoninka et al. 2011), leaving the climate response pattern open. Therefore, a unified conceptual framework of climate-driven plant-AM fungal community response - that incorporates community responses driven by abiotic forces while recognizing the mutualistic nature of mycorrhizal association that makes their community response unique-is needed to describe the response patterns between the symbionts.

Model projections show increasing impacts of climate extremes in the future, especially for extreme drought, which is predicted to increase in frequency and magnitude due to global climate warming (IPCC 2014; Xu et al . 2019). Many studies have reported diverse impacts of drought on both plant and soil microbial communities (de Vries et al. 2018; Luo et al. 2018, 2020); however, extreme drought is difficult to study due to its rarity and diverse occurrence patterns. Thus, field experiments taking into consideration of historical climate records and occurrence patterns are needed for extreme drought studies. For the plant-AM fungal symbionts, droughtinduced unilateral responses will finally feedback on plant-AM fungal community responses through the carbon-nutrient exchange. In this light, the primary pathway through which plants interact with AM fungal communities is not plant diversity and community structure, but rather plant productivity (Wagg et al. 2011; Powell \& Rillig 2018). Therefore, AM fungal diversity-plant productivity relationship may hold the key in plant-AM fungal community responses to climate change, yet reliable evidence under natural conditions is 
scarce.

To address this knowledge gap, we designed an in situ extreme drought experiment in a cold-temperate grassland with two types of rainfall manipulations: chronic and intense drought. For the chronic drought, we reduced the growing season rainfall by $66 \%$ from May to August; and for the intense drought, we suspended $100 \%$ rainfall from June to July (Fig. 1a; Fig. S2). Both types of drought reduced roughly $50 \%$ of mean annual precipitation, and the performance (probability of occurrence around $5 \%$ ) meet the criteria of climate extremes (Smith 2011; Slette et al . 2020) (Fig. 1a, b). Besides, we also set recovery treatments after drought to study the ecosystem resilience. The study was aimed to test the diversity-diversity and diversityproductivity response patterns between AM fungi and plant communities exposed to extreme drought. We hypothesized that climate-induced AM fungal diversity shifts will be positively correlated with plant diversity and productivity which may vary with climate conditions (i.e. chronic and intense drought). Furthermore, we predict that the combined effects of biotic and abiotic factors will be a strong predictor for soil AM fungal community responses.

\section{MATERIAL AND METHODS}

\section{Study site and experimental design}

This experiment was set up at the Erguna Forest-Steppe Ecotone Research Station (50¹0’N, 119deg22'E) in northeast China. The climate of the research area belongs to the cold temperate continental monsoon climate, with a mean annual precipitation of $363 \mathrm{~mm}$ and a mean annual temperature of -2.45. The soil type at this site is classified to chernozem (US soil taxonomy classification) and rich in carbon and nitrogen. The dominant plant species are Carex duriuscula , Leymus chinensis , Artemisia frigida , Pulsatilla turczaninovii ,Stipa baicalensis , Cymbaria dahurica andCleistogenes squarrosa (Table. S1). The experimental grassland was used for hay harvesting before 2013, and since then it has been fenced to prevent human and livestock interference.

We set up five treatments with two types of extreme drought (Fig. S2): (1) Control; (2) Chronic drought (CHR) - 3 years of chronic drought; (3) Chronic drought with recovery (CHRR) - 2 years of chronic drought and 1 year recovery; (4) Intense drought (INT) - 3 years of intense drought; (5) Intense drought with recovery (INTR) - 2 years of intense drought and 1 year recover (Fig. S2b). For the chronic drought (CHR/CHRR), we imposed a $66 \%$ precipitation reduction from May to August throughout the growing season, whereas for the intense drought (INT/INTR), we reduced 100\% precipitation from June to July (Fig. 1; Fig. S2a). Both types of drought reduced about $50 \%$ of annual precipitation with low occurrence incidence (most of them $<$ $5 \%$ ) based on historical climate data (Fig. 1a, b). During 2015-2016, we conducted the extreme drought with 12 plots per type. In 2017 after 2-years of drought, for each drought type, we randomly suspended drought stress in half of the replicates to investigate ecosystem resilience after drought (Fig. S2b). In this way, by comparing the treatment effects with control, we intend to eliminate the confounding effects of inter-annual precipitation variability.

We set up the field experiment using a randomized block design with six replicates for each treatment. We followed the rainout shelter design developed by Yahdjian and Sala (2002) with some modifications to minimize the microclimate effect caused by the experimental facilities. We use high light-transparent polyethylene partial roofs (Beijing Plastics Research Institute, Beijing, China) to reduce the rainfall amount passively (Fig. S2a). The metal scaffold supports the partial roofs aboveground to minimize greenhouse effects by permitting free air exchange (Fig. S1a). Before experimental setup, we assessed the microclimate effect: the partial roof permits over 90\% light transmission without air and soil temperature changes (automatically gathered with sensors). To prevent water flow into the plot, we hydrologically isolated the plot by trenching $1 \mathrm{~m}$ deep around the plot and lined the trench with plastic films and metal sheets (Fig. S1b). In the $6 \times 6 \mathrm{~m}$ plot area, we selected the central $4 \times 4 \mathrm{~m}$ area for further research, with the surrounding $1 \mathrm{~m}$ area serving as a buffer zone (Fig. S1c). The experiment is part of the global Drought-Net research network 
in China (https://drought-net.colostate.edu/).

\section{Plant and soil analyses}

We collected all the soil samples at the end of August 2017 after the chronic drought. In each plot, five soil cores of $0-20 \mathrm{~cm}$ depth $(3.8 \mathrm{~cm}$ in diameter) were collected and pooled to generate one composite soil sample. Soil samples were placed into a sterile plastic bags and stored with ice bags in a portable cooler box before transfer to the laboratory for further processing. The soil auger was cleaned using tap water between plots and dried using wipes. Then, we homogenized the soil samples by passing through a $2 \mathrm{~mm}$-sieve and collected the roots (stored at -20) for measuring AM colonization. Finally, we divided the soil samples into three subsamples and stored them at room temperature (air dry), 4, and -80, respectively.

Fresh roots were first cleaned using tap water and cut into $\sim 1 \mathrm{~cm}$ long fragments. We cleared the roots using $10 \% \mathrm{KOH}(25 \mathrm{~min}, 90)$, rinsed in $2 \% \mathrm{HCl}(5 \mathrm{~min})$, then stained in $0.05 \%$ trypan blue $(30 \mathrm{~min}, 70)$, and finally destained using lactic glycerol solution. Thirty root fragments per plot were randomly selected for microscopic inspection according to Trouvelot's method at magnification x200 (Trouvelot et al. 1986). The mycorrhizal colonization intensity (M\%) and the abundance of arbuscules were calculated using MYCOCALC software (https://www2.dijon.inrae.fr/mychintec/Mycocalc-prg/download).

The air-dried soil samples were ground using a ball mill (Retsch, MM400, Germany) and passed through $0.15 \mathrm{~mm}$-sieve for further analysis. We used a soil elemental analyzer (Vario EL III, Elementar, Germany) to measure soil total carbon and total nitrogen. Soil $\mathrm{pH}$ was measured using a soil to water ratio of 1:2.5 with a digital $\mathrm{pH}$ meter (FE200, Mettler Toledo, USA). Soil available P was measured following the Olsen method (Olsen 1954). Soil moisture was measured monthly using the gravimetric method (oven-dried (105) to constant weight), and the mean soil moisture content of each plot (from May to August) in 2017 was used for subsequent data analysis.

In August 2017, we collected the aboveground biomass of each species by clipping each plant at the ground level in a $1 \times 1 \mathrm{~m}^{2}$ area. We collected the root biomass $(0-20 \mathrm{~cm})$ using a root auger $(7 \mathrm{~cm}$ in diameter). Three soil cores were randomly collected within the $1 \times 1 \mathrm{~m}^{2}$ area used for plant investigation, and the roots were cleaned using tap water. All the roots were oven-dried (80) to a constant weight in the laboratory and weighed to calculate the belowground plant biomass in the $1 \times 1 \mathrm{~m}^{2}$ area by area conversion.

\section{DNA extraction and amplicon sequencing}

Soil DNA was extracted from $500 \mathrm{mg}$ freeze-dried soil samples using FastDNA ${ }^{\mathrm{TM}}$ SPIN Kit for Soil (MP Biomedicals, CA, USA) accompanied by Fastprep-24 5G sample homogenization (MP Biomedicals, CA, USA) following manufacturer's instructions. We used general fungal ITS primers, since they have been shown to have comparable effectiveness with AMF-specific SSU primers, and have the advantage to detect community-level responses across the fungal kingdom (Kohout et al . 2014; Berruti et al . 2017; Lekberg et al . 2018). The extracted DNA was then amplified using a barcoded primer set (fITS7: 5'GTGARTCATCGAATCTTTG-3'; ITS4: 5'-TCCTCCGCTTATTGATATGC-3') targeting the fungal ITS2 region (Ihrmarket al. 2012). Each of the $25 \mu \mathrm{l}$ PCR reaction system contained $12.5 \mu \mathrm{l} 2 \times$ Premix Taq (Takara Biotechnology, Dalian, China) and $2 \mu$ ldiluted DNA template. The PCR amplification was performed with an initial denaturation at $94^{\circ} \mathrm{C}$ for $5 \mathrm{~min}, 30$ cycles at $94^{\circ} \mathrm{C}$ for $30 \mathrm{~s}, 56^{\circ} \mathrm{C}$ for $30 \mathrm{~s}$, and $72^{\circ} \mathrm{C}$ for $30 \mathrm{~s}$ followed by $10 \mathrm{~min}$ extension at $72^{\circ} \mathrm{C}$. Three PCR replicates were performed for each sample and then pooled together to generate a composite PCR product. After PCR purification and pooling, sequencing libraries were constructed using NEBNext ${ }^{\circledR}$ Ultra $^{\text {TM }}$ DNA Library Prep Kit for Illumina (New England Biolabs, MA, USA) following the manufacturer's recommendations. Paired-end $(2 \times 250$ bases $)$ sequencing was performed using the Illumina Hiseq 2500 sequencing platform.

\section{Bioinformatics and statistical analyses}

DADA2 (1.12.1) was used for the bioinformatics analyses (Callahanet al. 2016). We used the ITS-specific version of the DADA2 workflow (1.8) for denoised ITS sequence variants (or amplicon sequence variants, ASVs (Callahanet al. 2017)) inference. Briefly, primers were first removed using cutadapt (Martin 2011); 
secondly, low-quality reads were filtered by filterAndTrim function; and then error rates were estimated for sample ASVs inference; next, chimeras were removed after merging paired reads; finally, taxonomy was assigned using the UNITE database (general release of all eukaryotes (2019.02.02)) (Kõljalget al. 2013). Overall, the DADA2 algorithm generated an ASV table of 30 samples $\times 6894$ ASVs $(2,169,792$ reads), of which 1669 ASVs were non-fungal (188,202 reads). Among all the fungal ASVs (1,981,590 reads), 489 ASVs $(15,814$ reads) were identified as AM fungi. We used two approaches to analyze soil AM fungal community responses. For the first approach (dataset1: traditional approach), to eliminate the effect of read differences on the following community composition analysis, we rarefied the AM fungal reads to 120 per sample. For the second approach (dataset2: rare species approach), we rarefied total fungal reads to 52,000 per sample to ensure an equal sampling depth. The second approach treats AM fungi as a subset of the fungal data (widely used in rare species studies), which eliminates the effects of different fungal reads and retained abundance variation of AM fungi among the fungal communities. We tested the sequencing efficiency using rarefaction curves (Fig. S3). The fungal rarefaction curve reached saturation around 15,000 reads per sample (Fig. S3a), suggesting 52,000 reads per sample was sufficient to cover almost all the fungal species. In line with this result, rarefaction curves of AM fungi in both dataset1 and dataset2 reached its plateau in almost all samples (Fig. S3b, c). Taken together, by deep ITS sequencing, we obtained sufficient reads to uncover the completeness of the AM fungal diversity in this experiment.

We analyzed the diversity of both datasets using the vegan $\mathrm{R}$ package (Oksanen et al. 2007). The two datasets showed consistent results in both $\alpha$ and $\beta$ diversity responses ( $\alpha$ diversity: Fig. S4 versus Fig. $2 b ; \beta$ diversity: Fig. S5a, b versus Fig. S5c, d), and also the response relationship with plant community (diversity-diversity (Fig. S6a versus Fig. $2 \mathrm{~d}$ ): $\mathrm{R}_{\text {dataset } 1}^{2}=0.21$ versus $\mathrm{R}^{2}$ dataset2 $=0.22$; diversity-productivity (Fig. S6b versus Fig. $2 \mathrm{e}$ ): $\mathrm{R}_{\text {dataset1 }}^{2}=0.24$ versus $\mathrm{R}_{\text {dataset2 }}^{2}=0.26$ ). As we used general fungal ITS primers, it is more reasonable to analyze community data using dataset 2 taking relative abundance variation into account, and compare AM fungal responses with other fungal groups. Therefore, we use dataset2 for the subsequent community response analysis (results of dataset1 could be found in SM).

General data manipulations and statistical analyses were performed in R (v3.60) (R Core Team 2013). The main $\mathrm{R}$ packages used in the data analyses are listed in Table S2, and detailed steps are described below and also in the code description.

Random forest (randomForest package, Liaw \& Wiener 2002) was used for quantifying the relative contribution of biotic and abiotic factors in predicting the responses of AM fungal richness to extreme drought, the $P$ -values were calculated using a one-sided binomial test as compiled in the randomForestExplainer $\mathrm{R}$ package (Paluszynskaet al. 2019). We used response ratio $\ln R R=\ln$ (Response per sample/Control mean) (Hedges et al. 1999) to normalize the responses of AM fungal richness, plant richness, and ANPP so that they can be compared directly. We employed unconstrained principal co-ordinates analysis (PCoA) to analyze AM fungal community shifts (Bray-Curtis dissimilarity), and significance was tested using PERMANOVA (adonis , vegan R package, Oksanen et al. 2007). Then, we used constrained (distance-based redundancy analysis, db-RDA) ordination to detect the main predictors of AM fungal community shifts, and the significance was tested using random permutations ( $\mathrm{n}=9999$ for all analyses). Given that AM fungal species could have multiple ITS ASVs (Thiéry et al. 2016; Bruns et al. 2018) and the high levels of ASV's resolution (Callahan et al. 2016), we performed the ordination analysis at both ASV-level and genus-level. Group comparisons were tested using the non-parametric Wilcoxon test.

The plant-AM fungi cross-kingdom network was constructed using Spearman correlation according to the method described by Ramirez et al. (2018) with some modifications. Given that network analysis is strongly influenced by the number of effective species (Inverse Simpson) and the sparsity of the species table (Weiss $e t$ al . 2016), we first filtered out all the species that appear less than 3 times among all samples. This approach lowered the sparsity of the combined species table with a reasonable number of effective species (12.8). Then we calculated Spearman correlation coefficients $(\rho)$ and $P$-values using corr.test function in the psych $\mathrm{R}$ package (William 2017), and the $P$-values were adjusted using the Benjamini-Hochberg method. Next, $P$ -values less than 0.01 were filtered. Finally, the correlation network was visualized using igraph package in 
R (Csardi \& Nepusz 2006).

\section{RESULTS}

\section{Synergistic response of AM fungal richness with plant richness and productivity}

Based on the denoised AM fungal ASV inference we successfully obtained 489 AM fungal ASVs belonging to 11 genera including Ambispora (1 ASV),Archaeospora (7 ASVs), Claroideoglomus (41 ASVs),Diversispora (14 ASVs), Dominikia (104 ASVs),Funneliformis (5 ASVs), Glomus (64 ASVs),Kamienskia (4 ASVs), Paraglomus (10 ASVs), Rhizophagus (55 ASVs), Septoglomus (35 ASVs) and eight unclassified genera (149 ASVs) (Fig. 2a). The overall AM fungal richness decreased significantly after 3-years of continuous drought (CHR/INT), however, recovered to the control level one year after the cessation of drought (CHRR/INTR) (Fig. 2b). The fungal richness did not significantly respond to drought, and at the fungal phylum level, only Glomeromycota showed a significant negative response (Fig. S7). Random forest analysis showed that the abrupt responses of AM fungal richness were best predicted by soil moisture, ANPP, and plant richness (Fig. 2c). Further correlation analyses revealed that responses of AM fungal richness were significantly and positively correlated with soil moisture $(P<0.001$; Fig. S8), plant richness $(P=0.0053$; Fig. 2 d $)$ and ANPP $(P=0.0023$; Fig. 2e). Moreover, the correlation coefficients differed between drought types. The adjusted $\mathrm{R}^{2}$ of the correlation between plant richness and AM fungal richness increased from $0.257(P>0.05, \mathrm{~ns})$ under chronic drought to $0.443(P<0.05)$ under intense drought (Fig. 2d); and the adjusted $\mathrm{R}^{2}$ of the ANPP and AM fungal richness boosted from $0.305(P<0.05)$ under chronic drought to 0.699 under intense drought $(P<0.001)$ (Fig. 2e). Finally, we normalized the responses of AM fungal ASVs richness, plant richness, and ANPP using response ratio for direct comparisons, and results showed a steadily consistent synergistic response pattern between the two types of extreme drought (Fig. 3).

\section{AM fungal community composition shifts with plant richness and productivity}

AM fungal community composition varied significantly after 3-years of continuous drought (CHR/INT), but the community composition recovered to control status after one-year recovery (CHRR and INTR) (Fig. S5). By using constrained ordination analysis (distance-based redundancy analysis, db-RDA), we showed that soil moisture, ANPP, and plant richness were among the best predictors for AM fungal community shifts, whereas AM colonization and soil physicochemical properties do not (Fig. 4a). The subsequent variance partitioning analysis showed that the abiotic variables are the main driving force for AM fungal community shifts, however, with a great portion ( $>40 \%$ ) of effect through the plant community (Fig. $4 \mathrm{~b})$. Further regression analysis showed that ANPP (Adjusted $\mathrm{R}^{2}=0.38, P<0.001$; Fig. 4c) and plant richness (Adjusted $\mathrm{R}^{2}=0.23, P<0.01$; Fig. S9) were significantly correlated with the first axis of db-RDA, which explained 16.8\% $(P<0.001)$ variance of AM fungal community shifts. Notably, correlation coefficient of $\operatorname{ANPP}\left(\mathrm{R}^{2}\right.$ chronic $=0.15$ to $\mathrm{R}^{2}$ intense $\left.=0.73\right)$ and plant richness $\left(\mathrm{R}^{2}\right.$ chronic $=0.12$ to $\left.\mathrm{R}^{2}{ }_{\text {intense }}=0.38\right)$ with db-RDA1 markedly increased from chronic drought to intense drought.

\section{Plant-AM fungi cross-kingdom correlation network}

To investigate intra- and inter-kingdom relationships of plant and AM fungi, we used Spearman correlations to perform taxon-specific network analysis. The integrated correlation network included 179 predicted nonrandomly distributed biotic edges among 41 plant species (species productivity), and 489 AM fungal ASVs (Fig. 5a; Table S4). The plant-AM fungi network showed a strong inter-kingdom correlation between plant species productivity and AM fungal ASVs (Fig. 5a). Specifically, most of the inter-kingdom correlations were limited to AM fungal genus Dominikia, an unclassified genus of Glomeraceae, Glomus , Rhizophagus ,Claroideoglomus, andDiversispora, most of which (except Diversispora) belong to Glomerales(Fig. 5b). For the plant community, Artemisia dracunculus, Calamagrostis angustifolia, Cleistogenes squarrosa, Potentilla acaulis, andPotentilla verticillaris have the most inter-kingdom edges with AM fungi (Table S3). The correlation network also contains intra-kingdom edges within plant species or AM fungal ASVs. For example, AM fungal ASVs within genus Dominikia, Glomus, Septoglomus, and Diversispora, and plant species of Cleistogenessquarrosa, Pulsatilla turczaninovii have most intra-kingdom edges in the network. Notably, ASVs within the AM fungal genus Septoglomus, Paraglomus only have intra-kingdom edges. 


\section{DISCUSSION}

Despite the fundamental importance of the mycorrhizal association in driving plant and AM fungal community ecology (van der Putten 2017; Neuenkampet al. 2018; Tedersoo et al. 2020), it remains unclear how the community responses of plant and AM fungi to climate change are interrelated. In this study based on a grassland extreme drought experiment, we show that: (1) abrupt changes of AM fungal richness are significantly and positively correlated with plant richness and productivity; (2) plant richness and productivity, together with soil moisture, were the best predictors of soil AM fungal community composition shifts; (3) both the robustness of plant-AM fungal association and the predictability of AM fungal community shifts by plant community increased with drought intensity; (4) Glomerales plays an important role in the interaction between plants and AM fungi, which largely drives the cross-kingdom interaction. Our findings suggest that the synergistic process between aboveground and belowground components may play key roles in shaping grassland plant and AM fungal biodiversity patterns following climate change.

The AM symbiosis can date back 450 Myr ago (Ma) (Brundrett \& Tedersoo 2018), and likely helped plants to adapt to the early harsh environments of terrestrial ecosystems (Wang et al. 2020). Within the mutualism, plant carbon is the only energy source for the fungus, which is usually constrained by plant photosynthesis linked to plant aboveground biomass. Thus, the interplay evolved between plants and AM fungi, mainly the carbon-nutrient exchange (Kiers et al. 2011), could explain our findings that responses of plant productivity and AM fungal community was positively associated (Fig. 2e). Subsequently, the symbiotic association would further influence community responses of both partners within a sufficient temporal scale (Chomicki et al.2019). In our case, a positive correlation between plant and AM fungal richness was observed after three years of treatment (Fig. 2d). The cooperation mechanism has already been addressed in greenhouse studies and may play key roles in developing diversity-diversity relationships between plants and AM fungi. For example, plants preferentially allocate more photosynthate to the more beneficial AM fungal partners (Beveret al. 2009); in turn, AM fungi would trade more phosphorus for carbon from plants when alternative AM fungi competitors are present (Argüello et al. 2016). The interplay between plants and AM fungi will finally feedback on the diversity of both partners. Empirical evidence of plant-AM fungi diversity correlations was also observed at large spatial scales in natural ecosystems (Öpik et al. 2009; Lekberg et al. 2013; Xuet al. 2016; Davison et al. 2020). For instance, in a 5000-km grassland transect in northern China, plant community composition was found to be a key predictor of AM fungal community shifts (Xu et al. 2016). This large scale investigation is consistent with our findings that plant richness and productivity were the best predictors for AM fungal community composition shifts other than soil moisture (Fig. 4). However, how the carbon-phosphorus exchange regulates the synergistic response of plant and AM fungal community to climate changes remains largely unknown.

It is universally acknowledged that environmental factors play a crucial role in shaping both plants and AM fungal communities (Franklin et al. 2016; Vályi et al. 2016; Deveautour et al. 2020). In line with these previous studies, our results also showed that soil moisture has a significant effect on plant and AM fungal diversity (Fig. 4; Fig S8). Interestingly, Glomeromycota is the only fungal phylum that showed significant negative responses to drought (Fig. S7). Beyond the abiotic forces, our results also showed a strong influence of plant factors (Fig. 2; Fig. 3; Fig. 4), which frames the plant-AM fungal community synergistic response. This finding differs from some previous empirical and theoretical studies highlighting that AM fungi drive plant biodiversity or vice versa (van der Heijden et al. 1998; van der Putten 2017). Instead, we argue that plant and AM fungal communities may respond cooperatively to grassland extreme drought. This inconsistency could be caused by the experimental setup and the spatial and temperal scale of the research (Vályi et al. 2016; Hempel 2018). Previously, plant-AM fungi interaction studies usually control one partner to investigate its effect on the other, which neglected dynamic community changes in both partners, and which may thus not be applicable to predict climate-driven community dynamics in natural ecosystems. Likewise, large scale investigations may suffer from biogeographical constraints (Veresoglou et al . 2019), environmental heterogeneity (Horn et al. 2017), and diversified community successional stages (Gao et al. 2019), which could mask plant-AM fungal community interactions (e.g. at regional scales (Van Geel et 
al. 2018) or global scales (Öpiket al. 2010)). Besides, plant and AM fungal community succession could vary at different temporal scales. For instance, AM fungi could show a strong community succession within weeks (Gao et al. 2019), whereas plants may take years (Hoover et al . 2014). For extreme events such as extreme drought, plants may be poorly prepared to respond at the community level, but its AM fungal partners may respond rapidly as a compensation. This hypothesis may explain that the robustness of this community association increases with drought intensity (Fig 2d, e; Fig 4c), perhaps indicating intensified mutual interdependence between plants and AM fungi.

The sensitivity of individual species to drought may also influence community responses (Engelbrecht et al. 2007; Ochoa-Hueso et al. 2018). Specifically, drought-induced growth, reproduction, and mortality responses may differ between plants and AM fungal species, and also between drought treatments, thereby inducing local response inconsistencies. This mechanism may also explain the variation of the association robustness from chronic to intense drought (Fig 2d, e; Fig 4c). For plant and AM fungal species, stress tolerance could be increased by their symbiotic association (Smithet al. 2009). Specifically, if we take mycorrhizal colonization as an indicator of plant-AM fungi interdependence, then both partners may benefit more from the intensified mycorrhizal colonization under drought. This may partially explain our results that, compared with INT treatment, AM fungi has a lower drought sensitivity under CHR treatment (Fig. 3), where mycorrhizal colonization was significantly intensified (Fig. S10).

Co-occurrence network analysis has rarely been applied between plant and AM fungal species. By doing so, we detected a strongly cross-kingdom network between plant species (productivity) and the AM fungal ASVs in response to extreme drought (Fig. 5). This result suggests that synergistic community response of plant-AM fungi occurs not only at the community level, but also take place at the species level. Given that AM fungal functions show both phylogenetic conservatism within AM fungal clades and differentiation among AM fungal clades (Maherali \& Klironomos 2007; Yang et al. 2017), it is highly likely that different AM fungal clades interact differently with plants. This concept might explain why the integrated network showed a non-random distribution pattern, with most of the inter-kingdom edges derived from Glomerales. Previous synthetic studies showed that species from Glomerales (e.g., Glomus deserticola, Claroideoglomus etunicatum ) had the largest average effects on plant drought resistance (Augé et al. 2015). The overrepresentation of interkingdom edges within Glomerales in our network may indicate that this AM fungal clade served more functions for plants under drought. In contrast, AM fungal ASVs inSeptoglomus, Paraglomus, and an unclassified genus of Diversisporales only have intrakingdom edges. This absence of abundance-productivity relationships of these AM fungal genera with plants may suggest that they interact differently with plants or lack of competitiveness with other genera. For example, introduction of non-native Rhizophagus irregularis could outcompete native AM fungal communities composed of Diversispora, Septoglomus and Paraglomus species (Symanczik et al . 2015). Nevertheless, no study has yet compared the interactiveness of different AM fungal clades with plants, it is too early to speculate the interaction patterns through the plant-AM fungal correlation network.

Climate change is imposing an enormous threat to natural ecosystems and has caused substantial alterations of biodiversity patterns across ecosystems (Bellard et al. 2012). Integrating and disentangling the interplay between aboveground plants and belowground microbes remains an essential step to understand the ecological impacts of climate change. Arbuscular mycorrhizal fungi, an ancient phylum of fungi forming mutualistic symbioses with the majority of land vascular plants (Brundrett \& Tedersoo 2018), hold enormous significance for an integrated ecosystem. Despite the limited spatial scale involved, our findings clearly uncovered climate change-induced diversity-diversity and productivity-diversity response patterns between plants and AM fungi. Such relationships may underpin climate change impact on grassland biodiversity patterns. With the predicted increasing frequency and severity of climate extremes worldwide, generalized patterns will be urgently needed for explicitly modeling the ecological impacts.

Acknowledgements : We thank all the staff in Erguna Forest-Steppe Ecotone Research Station for their 
help during the experiment. This research was supported by National Key Research and Development Program of China (2016YFC0500702). The authors declare no conflict of interest.

\section{REFERENCES:}

Antoninka, A., Reich, P.B. \& Johnson, N.C. (2011). Seven years of carbon dioxide enrichment, nitrogen fertilization and plant diversity influence arbuscular mycorrhizal fungi in a grassland ecosystem. New Phytol. , 192, 200-214.

Argüello, A., O’Brien, M.J., van der Heijden, M.G.A., Wiemken, A., Schmid, B. \& Niklaus, P.A. (2016). Options of partners improve carbon for phosphorus trade in the arbuscular mycorrhizal mutualism.Ecol. Lett. , 19, 648-656.

Augé, R.M., Toler, H.D. \& Saxton, A.M. (2015). Arbuscular mycorrhizal symbiosis alters stomatal conductance of host plants more under drought than under amply watered conditions: a meta-analysis. Mycorrhiza , 25, 13-24.

Bardgett, R.D. \& van der Putten, W.H. (2014). Belowground biodiversity and ecosystem functioning. Nature , 515, 505-511.

Bellard, C., Bertelsmeier, C., Leadley, P., Thuiller, W. \& Courchamp, F. (2012). Impacts of climate change on the future of biodiversity. Ecol. Lett. , 15, 365-377.

Bever, J.D., Richardson, S.C., Lawrence, B.M., Holmes, J. \& Watson, M. (2009). Preferential allocation to beneficial symbiont with spatial structure maintains mycorrhizal mutualism. Ecol. Lett., 12, 13-21.

Berruti, A., Desirò, A., Visentin, S., Zecca, O. \& Bonfante, P. (2017). ITS fungal barcoding primers versus 18S AMF-specific primers reveal similar AMF-based diversity patterns in roots and soils of three mountain vineyards. Env. Microbiol. Rep. , 9, 658-667.

Bowles, T.M., Jackson, L.E. \& Cavagnaro, T.R. (2018). Mycorrhizal fungi enhance plant nutrient acquisition and modulate nitrogen loss with variable water regimes. Global Change Biol., 24, e171-e182.

Brundrett, M.C. \& Tedersoo, L. (2018). Evolutionary history of mycorrhizal symbioses and global host plant diversity. New Phytol. , 220, 1108-1115.

Bruns, T.D., Corradi, N., Redecker, D., Taylor, J.W. \& Öpik, M. (2018). Glomeromycotina: What is a species and why should we care? New Phytol. , 220, 963-967.

Callahan, B.J., McMurdie, P.J. \& Holmes, S.P. (2017). Exact sequence variants should replace operational taxonomic units in marker-gene data analysis. ISME J. , 11, 2639-2643.

Callahan, B.J., McMurdie, P.J., Rosen, M.J., Han, A.W., Johnson, A.J. \& Holmes, S.P. (2016). DADA2: high-resolution sample inference from Illumina amplicon data. Nat. Methods , 13, 581-583.

Chomicki, G., Weber, M., Antonelli, A., Bascompte, J. \& Kiers, E.T. (2019). The impact of mutualisms on species richness. Trends Ecol. Evol. , 34, 698-711.

Csardi, G. \& Nepusz, T. (2006). The igraph software package for complex network research. Inter. J. Comp. Syst. , 1695, 1-9.

Davison, J., Moora, M., Öpik, M., Adholeya, A., Ainsaar, L., Bâ, A.et al. (2015). Global assessment of arbuscular mycorrhizal fungus diversity reveals very low endemism. Science , 349, 970-973.

Davison, J., García de León, D., Zobel, M., Moora, M., Bueno, C.G., Barceló, M. et al. (2020). Plant functional groups associate with distinct arbuscular mycorrhizal fungal communities. New Phytol. , 226, $1117-1128$.

Delavaux, C.S., Smith-Ramesh, L.M. \& Kuebbing, S.E. (2017). Beyond nutrients: a meta-analysis of the diverse effects of arbuscular mycorrhizal fungi on plants and soils. Ecology , 98, 2111-2119. 
Deveautour, C., Power, S.A., Barnett, K.L., Ochoa-Hueso, R., Donn, S., Bennett, A.E. et al. (2020). Temporal dynamics of mycorrhizal fungal communities and co-associations with grassland plant communities following experimental manipulation of rainfall. J. Ecol. , 108, 515-527.

de Vries, F.T., Griffiths, R.I., Bailey, M., Craig, H., Girlanda, M., Gweon, H.S. et al. (2018). Soil bacterial networks are less stable under drought than fungal networks. Nat. Commun., 9, 3033.

Engelbrecht, B.M.J., Comita, L.S., Condit, R.S., Kursar, T.A., Tyree, M.T., Turner, B.L. et al. (2007). Drought sensitivity shapes species distribution patterns in tropical forests. Nature, 447, 80-82.

Hempel, S. (2018). Passengers and drivers of arbuscular mycorrhizal fungal communities at different scales. New Phytol. , 220, 952-953.

Franklin, J., Serra-Diaz, J.M., Syphard, A.D. \& Regan, H.M. (2016). Global change and terrestrial plant community dynamics. P. Natl. Acad. Sci. USA , 113, 3725-3734.

Gao, C., Montoya, L., Xu, L., Madera, M., Hollingsworth, J., Purdom, E.et al. (2019). Strong succession in arbuscular mycorrhizal fungal communities. ISME J. , 13, 214-226.

Götzenberger, L., de Bello, F., Bråthen, K.A., Davison, J., Dubuis, A., Guisan, A. et al. (2012). Ecological assembly rules in plant communities-approaches, patterns and prospects. Biol. Rev. , 87, 111-127.

Hart, M.M., Reader, R.J. \& Klironomos, J.N. (2001). Life-history strategies of arbuscular mycorrhizal fungi in relation to their successional dynamics. Mycologia , 93, 1186-1194.

Hedges, L.V., Gurevitch, J. \& Curtis, P.S. (1999). The meta-analysis of response ratios in experimental ecology. Ecology , 80, 1150-1156.

Hiiesalu, I., Pärtel, M., Davison, J., Gerhold, P., Metsis, M., Moora, M. et al. (2014). Species richness of arbuscular mycorrhizal fungi: associations with grassland plant richness and biomass. New Phytol. , 203, 233-244.

Hoover, D.L., Knapp, A.K. \& Smith, M.D. (2014). Resistance and resilience of a grassland ecosystem to climate extremes. Ecology , 95, 2646-2656.

Horn, S., Hempel, S., Verbruggen, E., Rillig, M.C. \& Caruso, T. (2017). Linking the community structure of arbuscular mycorrhizal fungi and plants: a story of interdependence? ISME J. , 11, 1400-1411.

Ihrmark, K., Bodeker, I.T., Cruz-Martinez, K., Friberg, H., Kubartova, A., Schenck, J. et al. (2012). New primers to amplify the fungal ITS2 region evaluation by 454-sequencing of artificial and natural communities. FEMS Microbiol. Ecol. , 82, 666-677.

IPCC (2014).Climate change 2014: synthesis report. Contribution of working groups I, II, and III to the fifth assessment report of the Intergovernmental Panel on Climate Change. Cambridge University Press, Cambridge, UK.

Karlowsky, S., Augusti, A., Ingrisch, J., Hasibeder, R., Lange, M., Lavorel, S. et al. (2018). Land use in mountain grasslands alters drought response and recovery of carbon allocation and plant-microbial interactions. J. Ecol. , 106, 1230-1243.

Kiers, E.T., Duhamel, M., Beesetty, Y., Mensah, J.A., Franken, O., Verbruggen, E. et al. (2011). Reciprocal rewards stabilize cooperation in the mycorrhizal symbiosis. Science, 333, 880-882.

Kohout, P., Sudová, R., Janoušková, M., Čtvrtlíková, M., Hejda, M., Pánková, H. et al. (2014). Comparison of commonly used primer sets for evaluating arbuscular mycorrhizal fungal communities: is there a universal solution? Soil Biol. Biochem., 68, 482-493.

Kõljalg, U., Nilsson, R.H., Abarenkov, K., Tedersoo, L., Taylor, A.F.S., Bahram, M. et al. (2013). Towards a unified paradigm for sequence-based identification of fungi. Mol. Ecol. , 22, 5271-5277. 
Lekberg, Y., Gibbons, S.M., Rosendahl, S. \& Ramsey, P.W. (2013). Severe plant invasions can increase mycorrhizal fungal abundance and diversity.ISME J. , 7, 1424-1433.

Lekberg, Y., Vasar, M., Bullington, L.S., Sepp, S.-K., Antunes, P.M., Bunn, R. et al. (2018). More bang for the buck? Can arbuscular mycorrhizal fungal communities be characterized adequately alongside other fungi using general fungal primers? New Phytol. , 220, 971-976.

Liaw, A. \& Wiener, M. (2002). Classification and regression by randomForest. $R$ news , 2, 18-22.

Li, T., Hu, Y.J., Hao, Z.P., Li, H., Wang, Y.S. \& Chen, B.D. (2013). First cloning and characterization of two functional aquaporin genes from an arbuscular mycorrhizal fungus Glomus intraradices .New Phytol. , 197, 617-630.

Luo, W.T., Zuo, X.A., Ma, W., Xu, C., Li, A., Yu, Q. et al. (2018). Differential responses of canopy nutrients to experimental drought along a natural aridity gradient. Ecology , 99, 2230-2239.

Luo, W.T., Zuo, X.A., Griffin-Nolan, R.J., Xu, C., Sardans, J., Yu, Q. et al. (2020). Chronic and intense droughts differentially influence grassland carbon-nutrient dynamics along a natural aridity gradient.Plant Soil , https://doi.org/10.1007/s11104-020-04571-8.

Maherali, H. \& Klironomos, J.N. (2007). Influence of phylogeny on fungal community assembly and ecosystem functioning. Science, 316, 1746-1748.

Martin, M. (2011). Cutadapt removes adapter sequences from high-throughput sequencing reads. EMBnet. J. , 17, 10-12.

Neuenkamp, L., Moora, M., Opik, M., Davison, J., Gerz, M., Mannisto, M.et al. (2018). The role of plant mycorrhizal type and status in modulating the relationship between plant and arbuscular mycorrhizal fungal communities. New Phytol. , 220, 1236-1247.

Ochoa-Hueso, R., Collins, S.L., Delgado-Baquerizo, M., Hamonts, K., Pockman, W.T., Sinsabaugh, R.L. et al. (2018). Drought consistently alters the composition of soil fungal and bacterial communities in grasslands from two continents. Global Change Biol. , 24, 2818-2827.

Oksanen, J., Kindt, R., Legendre, P., O'Hara, B., Stevens, M.H.H., Oksanen, M.J. et al. (2007). vegan: community ecology package. Available at: https://cran.r-project.org/web/packages/vegan/index.html. Last accessed 25 April 2020.

Olsen, S.R. (1954). Estimation of available phosphorus in soils by extraction with sodium bicarbonate. US Department of Agriculture.

Öpik, M., Metsis, M., Daniell, T.J., Zobel, M. \& Moora, M. (2009). Large-scale parallel 454 sequencing reveals host ecological group specificity of arbuscular mycorrhizal fungi in a boreonemoral forest. New Phytol. , 184, 424-437.

Öpik, M., Vanatoa, A., Vanatoa, E., Moora, M., Davison, J., Kalwij, J.M.et al. (2010). The online database MaarjAM reveals global and ecosystemic distribution patterns in arbuscular mycorrhizal fungi (Glomeromycota). New Phytol. , 188, 223-241.

Paluszynska, A., Biecek, P., Jiang, Y. (2019). randomForestExplainer: Explaining and visualizing random forests in terms of variable importance. Available at: https://cran.rproject.org/web/packages/randomForestExplainer/index.html. Last accessed 25 April 2020.

Powell, J.R. \& Rillig, M.C. (2018). Biodiversity of arbuscular mycorrhizal fungi and ecosystem function. New Phytol. , 220, 1059-1075.

Ramirez, K.S., Geisen, S., Morriën, E., Snoek, B.L. \& van der Putten, W.H. (2018). Network analyses can advance above-belowground ecology. Trends Plant Sci. , 23, 759-768. 
R Core Team, R. (2013). R: a language and environment for statistical computing. R foundation for statistical computing, Vienna, Austria.

Rillig, M.C., Wendt, S., Antonovics, J., Hempel, S., Kohler, J., Wehner, J. et al. (2014). Interactive effects of root endophytes and arbuscular mycorrhizal fungi on an experimental plant community. Oecologia, 174, 263-270.

Ruiz-Lozano, J.M., Aroca, R., Zamarreño, Á.M., Molina, S., Andreo-Jiménez, B., Porcel, R. et al. (2016). Arbuscular mycorrhizal symbiosis induces strigolactone biosynthesis under drought and improves drought tolerance in lettuce and tomato. Plant Cell Environ. , 39, 441-452.

Slette, I.J., Smith, M.D., Knapp, A.K., Vicente-Serrano, S.M., Camarero, J.J. \& Beguería, S. (2020). Standardized metrics are key for assessing drought severity. Global Change Biol. , 26, e1-e3.

Smith, M.D. (2011). An ecological perspective on extreme climatic events: a synthetic definition and framework to guide future research.J. Ecol. , 99, 656-663.

Smith, S.E., Facelli, E., Pope, S. \& Andrew Smith, F. (2009). Plant performance in stressful environments: interpreting new and established knowledge of the roles of arbuscular mycorrhizas. Plant Soil , 326, 3-20.

Smith, S.E. \& Read, D.J. (2010). Mycorrhizal symbiosis, Third Edition . Academic Press, London, UK.

Symanczik, S., Courty, P.E., Boller, T., Wiemken, A. \& Al-Yahya'ei, M.N. (2015). Impact of water regimes on an experimental community of four desert arbuscular mycorrhizal fungal (AMF) species, as affected by the introduction of a non-native AMF species. Mycorrhiza , 25, 639-647.

Tedersoo, L., Bahram, M. \& Zobel, M. (2020). How mycorrhizal associations drive plant population and community biology.Science, 367, eaba1223.

Thiéry, O., Vasar, M., Jairus, T., Davison, J., Roux, C., Kivistik, P.A.et al. (2016). Sequence variation in nuclear ribosomal small subunit, internal transcribed spacer and large subunit regions of Rhizophagus irregularis and Gigasporamargarita is high and isolate-dependent. Mol. Ecol. , 25, 2816-2832.

Trouvelot A., Kough J.L., Gianinazzi-Pearson V. (1986). Mesure du taux de mycorhization VA d'un système radiculaire. Recherche de méthodes d'estimation ayant une signification fonctionnelle. In: Physiological and genetical aspects of mycorrhizae. V. Gianinazzi-Pearson and S. Gianinazzi (eds.). INRA Press, Paris, pp. $217-221$.

Vályi, K., Mardhiah, U., Rillig, M.C. \& Hempel, S. (2016). Community assembly and coexistence in communities of arbuscular mycorrhizal fungi.ISME J. , 10, 2341-2351.

van der Heijden, M.G.A., Klironomos, J.N., Ursic, M., Moutoglis, P., Streitwolf-Engel, R., Boller, T. et al. (1998). Mycorrhizal fungal diversity determines plant biodiversity, ecosystem variability and productivity. Nature , 396, 69-72.

van der Putten, W.H. (2017). Belowground drivers of plant diversity.Science, 355, 134-135.

Vandenkoornhuyse, P., Quaiser, A., Duhamel, M., Le Van, A. \& Dufresne, A. (2015). The importance of the microbiome of the plant holobiont.New Phytol. , 206, 1196-1206.

Van Geel, M., Jacquemyn, H., Plue, J., Saar, L., Kasari, L., Peeters, G.et al. (2018). Abiotic rather than biotic filtering shapes the arbuscular mycorrhizal fungal communities of European seminatural grasslands. New Phytol. , 220, 1262-1272.

Veresoglou, S.D., Liu, L., Xu, T.L., Rillig, M.C., Wang, M.E., Wang, J.T. et al. (2019). Biogeographical constraints in Glomeromycotinan distribution across forest habitats in China. J. Ecol. , 107, 684-695.

Wagg, C., Jansa, J., Schmid, B. \& van der Heijden, M.G.A. (2011). Belowground biodiversity effects of plant symbionts support aboveground productivity. Ecol. Lett. , 14, 1001-1009. 
Wang, S.H., Guan, Y.L., Wang, Q., Zhao, J.J., Sun, G.L., Hu, X.Y.et al. (2020). A mycorrhizae-like gene regulates stem cell and gametophore development in mosses. Nat. Commun. , 11, 2030.

Weiss, S., Van Treuren, W., Lozupone, C., Faust, K., Friedman, J., Deng, Y. et al. (2016). Correlation detection strategies in microbial data sets vary widely in sensitivity and precision. ISME J. , 10, 1669-1681.

William R. (2017). Psych: procedures for psychological, psychometric, and personality research. Available at: https://cran.r-project.org/web/packages/psych/index.html. Last accessed 25 April 2020.

Xu, C.G., McDowell, N.G., Fisher, R.A., Wei, L., Sevanto, S., Christoffersen, B.O. et al. (2019). Increasing impacts of extreme droughts on vegetation productivity under climate change. Nat. Clim. Change, 9, 948953.

Xu, L.J., Li, T., Wu, Z.X., Feng, H.Y., Yu, M., Zhang, X. et al. (2018). Arbuscular mycorrhiza enhances drought tolerance of tomato plants by regulating the 14-3-3 genes in the ABA signaling pathway. Appl. Soil Ecol. , 125, 213-221.

Xu, T.L., Veresoglou, S.D., Chen, Y.L., Rillig, M.C., Xiang, D., Ondřej, D. et al. (2016). Plant community, geographic distance and abiotic factors play different roles in predicting AMF biogeography at the regional scale in northern China. Env. Microbiol. Rep. , 8, 1048-1057.

Yahdjian, L. \& Sala, O.E. (2002). A rainout shelter design for intercepting different amounts of rainfall. Oecologia , 133, 95-101.

Yang, H.S., Zhang, Q., Koide, R.T., Hoeksema, J.D., Tang, J.J., Bian, X.M. et al. (2017). Taxonomic resolution is a determinant of biodiversity effects in arbuscular mycorrhizal fungal communities.J. Ecol. , 105, 219-228.

Yang, G.W., Wagg, C., Veresoglou, S.D., Hempel, S. \& Rillig, M.C. (2018). How soil biota drive ecosystem stability. Trends Plant Sci. , 23, 1057-1067.

Zobel, M. \& Öpik, M. (2014). Plant and arbuscular mycorrhizal fungal (AMF) communities-which drives which? J. Veg. Sci. , 25, 1133-1140.

Figures

Figure 1 Experimental design of the grassland extreme drought experiment. (a ) Experimental design. During the growing season of 2015-2017, the chronic and intense droughts were implemented by reducing the ambient rainfall using light-transparent partial roofs. The inset shows both drought types have similar annual mean precipitation based on historical precipitation data. (b ) The performance of the experimental setup. The experimental treatment resulted in extreme drought in 2015, 2016, and 2017 mapping on the estimated probability density curve based on historical precipitation data. The annual mean precipitation and probability density curve were estimated using 61-year historical climate data from 19572017.

Figure 2 AM fungal alpha diversity responses and its associations with plant richness and aboveground net primary productivity (ANPP). (a ) Relative abundance of AM fungal genus in the fungal community of each treatment. (b ) The response of AM fungal ASVs (amplicon sequence variants) richness. The black points jittered around the boxplot represents the raw data (Wilcoxon test, ${ }^{*} P<0.05$, $\left.{ }^{* *} P<0.01\right)$. (c ) Random forest analysis showing the relative contribution of biotic and abiotic factors in predicting AM fungal ASVs richness response to extreme drought (the more important the index, the greater the values). Abiotic variables: soil moisture (2017), soil $\mathrm{C}, \mathrm{N}$ content, $\mathrm{C} / \mathrm{N}, \mathrm{pH}$, and available phosphorus content (AP); biotic variables: aboveground net primary productivity (ANPP), belowground biomass and plant richness. (d ) The response relationship of AM fungal ASVs richness and plant richness. (e ) The response relationship of AM fungal ASVs richness and ANPP. All the linear regressions fitted using the OLS model. CHR: chronic drought; CHRR: chronic drought with recovery; INT: intense drought; INTR: intense drought with recovery. 
Figure 3 Standardized response pattern of AM fungal richness, plant richness, and ANPP induced by the grassland extreme droughts. The filled dots and whiskers represent the mean response ratio and 95\% confidence intervals. AM fungal richness, plant richness, and ANPP response ratios (compared to control) were calculated using responses of AM fungal ASVs richness (red), plant richness (blue), and aboveground net primary productivity (ANPP; green), respectively. CHR: chronic drought; CHRR: chronic drought with recovery; INT: intense drought; INTR: intense drought with recovery.

Figure 4 AM fungal community response and its associations with plant community. (a ) Constrained ordination analysis (db-RDA at genus level) revealed the main factors responsible for the AM fungal community composition response (Permutation test $(\mathrm{n}=9999),{ }^{*} P<0.05,{ }^{* *} P<0.01$, ${ }^{* * *} P<$ 0.001). (b ) Variance partitioning analysis of plant and abiotic variables in predicting AM fungal community composition shifts (Permutation test $\left.(\mathrm{n}=9999),{ }^{*} P<0.05,{ }^{* *} P<0.01,{ }^{*} * * P<0.001\right)$. (c ) Regression analysis of ANPP and the constrained AM fungal community variation (db-RDA1). CHR: chronic drought; CHRR: chronic drought with recovery; INT: intense drought; INTR: intense drought with recovery.

Figure 5 Network analyses for exploring plant-AM fungi cross-kingdom correlation. (a ) PlantAM fungal cross-kingdom correlation network. Nodes represent AM fungal ASVs and plant species productivity; edges represent significant Spearman correlations $(P<0.01)$. Node size were weighted by the number of its edges. Gold lines represent interkingdom correlations; gray lines represent intrakingdom correlations. (b ) Edge distribution across AM fungal phylogenetic taxa.

\section{Hosted file}

Figures 201107.pdf available at https://authorea.com/users/378860/articles/495271synergistic-community-responses-of-plants-and-arbuscular-mycorrhizal-fungi-to-extremedroughts-in-a-cold-temperate-grassland 